\title{
A headway to improve PTW rider safety within the EU through three types of ITS

\author{
Anne Silla ${ }^{1 *}$, Lars Leden ${ }^{1}$, Pirkko Rämä ${ }^{1}$, Johan Scholliers ${ }^{1}$, Martijn van Noort ${ }^{2}$, Andrew Morris ${ }^{3}$, \\ Graham Hancox ${ }^{3}$ and Daniel Bell ${ }^{4}$
}

\begin{abstract}
Introduction: The current safety situation for Powered Two Wheelers (PTW) within the EU is alarming. According to EU statistics, PTW riders account for $17 \%$ of all fatal road injuries in the region and twice as many fatalities per hundred thousand registered vehicles compared with occupants of cars. In recent years, too little attention has been given to Vulnerable Road Users (VRUs), including PTW users, in the development of Intelligent Transport Systems (ITS). Therefore, ITS should be developed that consider PTW riders an integral part of traffic; we focus on three systems that we believe have the potential to improve safety among PTW riders, namely Intersection Safety (INS), the Powered Two Wheeler oncoming vehicle information system (PTW2V), and the Vulnerable road user Beacon System (VBS). The present study aims to estimate quantitatively the safety impacts of the selected ITS for PTW riders in the EU-28, once the systems are fully adopted and meet selected future scenarios for 2020 and 2030.

Method: An ex-ante method suggested by Kulmala (Accident Analysis and Prevention 42:1359-1369, 2010) was further developed and applied to assess the safety impacts of ITS specifically designed for VRUs. As suggested by the method, the analysis started by determining the impact mechanisms through which the selected ITS systems affect the safety of PTW riders.
\end{abstract}

Results: According to the main results, all the systems we studied have a positive impact on PTW rider safety by preventing fatalities and injuries. The greatest effects, based on 2012 accident data and full penetration, could be attained by implementing PTW2V (283 fewer yearly fatalities) and INS (261 fewer yearly fatalities). The weakest effect was found with VBS (216 fewer yearly fatalities). Forecasts for 2030, also based on estimated accident trends and penetration rates, confirm an expected edge for INS and PTW2V in terms of safety.

Keywords: Vulnerable road users, Intelligent transport system, Safety impact assessment, PTW riders

\section{Introduction}

The current safety situation for PTW riders within the EU is alarming. According to EU statistics [1], PTW riders account for $17 \%$ of all fatal road injuries in the region. Eleven PTW drivers or passengers are killed per 100,000 registered PTWs, compared with only five per same amount of registered cars [2]. The share of PTW rider fatalities among all road deaths varies widely among Member States, from $4 \%$ in Romania to $37 \%$ in Greece [1]. A factor contributing to this skewness is that in many areas PTW mobility is much higher than passenger vehicle mobility, especially in countries like

\footnotetext{
* Correspondence: anne.silla@vtt.fi

${ }^{1}$ VTT Technical Research Centre of Finland Ltd, P.O. Box 1000, 02044 VTT,

Espoo, Finland

Full list of author information is available at the end of the article
}

Greece, Italy and France with congested traffic conditions. Even in Sweden, 98\% of commuters regularly choosing to ride a motorcycle to and from work in good weather stated that one reason for commuting by PTW is to save time [3].

In order to highlight the safety hazards of Powered Two Wheelers (PTWs), the Driving Reliability and Error Analysis Method (DREAM) was used to classify the factors contributing to PTW crashes investigated in Finland, France and the United Kingdom as part of the EU project 2-be-safe. Below, only the results concerning motorcycles are described. Fatal rider injuries outside urban areas in three accident configurations (scenarios) were explored: single motorcycle crashes, crashes between motorcycles and passenger cars outside and at intersections. Concerning single motorcycle crashes, the 
most common human factors contributing to crashes are misjudgement of the situation due to inattention, late observation and insufficient skills. Factors related to the traffic environment include inadequate design and poor road maintenance and/or sight obstruction. In crashes between riders and passenger cars, the main factor concerning riders is misjudgement of the situation, mainly through expecting a certain behaviour from someone else, and outside intersections also priority errors and inadequate road design and sight obstruction. The main contributing factor for drivers is misjudgement of situations through expecting a certain behaviour, and inattention [4].

An analysis of Swedish in-depth crash data on fatal motorcycle rider injuries in 2005-2010 revealed even more serious shortcomings than did the 2-be-safe project: $25 \%$ of riders had no licence, $36 \%$ were speeding significantly, $26 \%$ were intoxicated by alcohol or drugs, and $7 \%$ wore no helmet [5]. Existing laws and regulations should enhance and ensure communication between road users, but in this case it seems not to be working. Apart from surveillance, riding with safety margins implies a concept that could be helpful also in more severe circumstances. One such concept could be to make motorcyclists and cars "visible" to each other, for example through warning lights, signs or messages within the infrastructure, in-vehicle alarms to warn of conflicting road users, or if necessary even the application of emergency braking.

Beanland et al. [6] assessed the acceptance of different assistive systems targeted to PTW riders. The results showed that systems having the highest overall rider acceptability within the countries taking part in the 2be-safe project were night vision, anti-lock braking system (ABS), advanced front-lighting system and eCall. The three lowest-ranked systems were Intelligent Speed Adaption (ISA), lane keeping assistant and adaptive cruise control.

A forgiving roadside is especially important for riders. In Sweden, 19 riders (11\% of all rider fatalities in the country) were fatally injured when hitting guard rails (barriers) in 2005-2008 [7]. Today, the barriers have been softened to reduce impact forces when hit. In the Norwegian design standards, seven of 18 issues on the checklist for rider safety concern barrier design [8].

Intelligent Transport Systems (ITS) have recently been effective in decreasing the number of road traffic fatalities, specifically among passenger car occupants. Vulnerable Road Users (VRUs), such as motorcyclists and moped riders, have received too little attention in the development of ITS (e.g. [9]). Consequently, there is a need for ITS that specifically address PTW riders as an integral part of traffic. We focus on three systems considered to have good potential for improving the safety of PTW riders: Intersection Safety (INS), the Powered Two Wheeler oncoming vehicle information system (PTW2V) and the Vulnerable road user Beacon System (VBS).

The study presented here aimed to quantitatively estimate the safety impacts of the selected ITS on PTW riders in the EU-28, once the systems are fully adopted in line with selected future scenarios in 2020 and 2030. An ex-ante assessment method has been suggested by Kulmala [10] for the traffic safety impacts of ITS. The method has been used in expert assessments of ITS for cars. The same approach was further developed and applied in this study to assess the safety impacts of ITS specifically designed for VRUs. As suggested by the method, the analysis started by determining the impact mechanisms through which the selected ITS systems affect the safety of PTW riders.

\section{Intelligent transport systems improving the safety of powered-two-wheeler riders}

\subsection{Selection of systems}

First, a list of 23 ITS was drawn up. This included all ITS deemed to be near to market and to have good overall potential to improve the safety, mobility and/or comfort of VRUs. Subsequently, the impacts of these systems on safety, mobility and comfort were assessed qualitatively [11], and based on this a subset of 10 ITS was selected for quantitative assessment. This selection was done in a workshop using a multi-criteria assessment and portfolio check (ex-post check on the overall result) [12]. The multi-criteria analysis ranked the systems, while the portfolio check determined whether all important aspects were covered. The multi-criteria selection included issues such as benefits, costs, deployment and users, and the portfolio check confirmed, for example, that the systems addressed all vulnerable road user groups, covered all impact categories, and covered different types of ITS (infra-based, car-based, VRU-based and cooperative ITS).

Four of these ten systems were targeted to PTW riders. Of the four, Blind Spot Detection (BSD) was only estimated to be effective in preventing moped accidents, not motorcyclist accidents. Therefore, the systems selected for the assessment presented here included Intersection Safety (INS), the Powered Two Wheeler oncoming information system (PTW2V) and the Vulnerable road user Beacon system (VBS). The systems are presented in greater detail in chapter 2.2.

\subsection{Description of systems}

A short description of each assessed system is included below, with figures illustrating their performance (Fig. 1). 


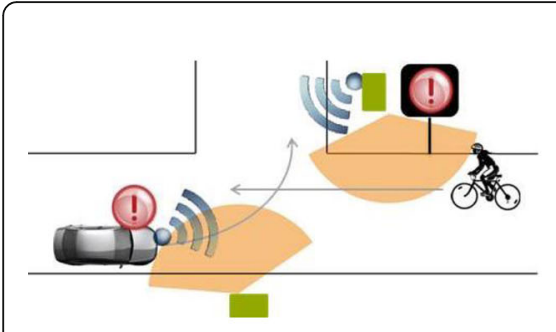

a) Intersection Safety (INS)

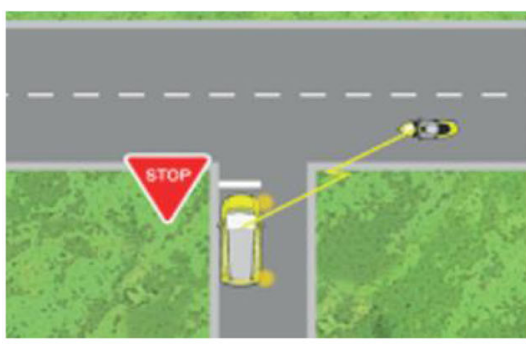

b) Powered Two Wheeler oncoming vehicle information systems (PTW2V)

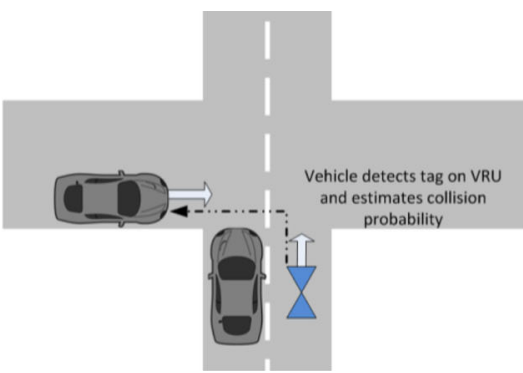

c) VRU Beacon System (VBS)

Fig. 1 Systems assessed. a Intersection Safety (INS). b Powered Two Wheeler oncoming vehicle information systems (PTW2V). c VRU Beacon System (VBS)

\subsubsection{Intersection safety (INS)}

The system assists the driver and VRU at intersections. It covers both conflicts between vehicles and VRUs during left- and right turning and vehicles arriving perpendicular to VRUs. Left- and right-turning assistance pertains to a vehicle turning left or right into the path of a VRU. A roadside unit (RSU) at an intersection detects conflicting VRUs, in our case a rider, via camera or radar, assesses the risk of collision, and sends a warning of potential collision to the vehicle. The vehicle driver is informed or receives a warning via an on-board unit. In addition, the RSU warns the VRU of the danger with, for example, flashing lights and/or sound. The system covers two functions, for left- and right-turning assistance and for conflicting vehicles arriving on perpendicular courses. Left- and right-turning assistance pertains to a vehicle turning left or right into the path of the rider. The process for conflicting vehicles with perpendicular courses is the same as for the first function: an RSU detects the rider crossing the intersection and informs the vehicle of a possible collision. The system depends on short-range communication and roadside sensors. Optionally, the vehicle also uses its own sensors. The system does not intervene; it aims to prevent accidents between cars/trucks/buses and VRUs at signalised and non-signalised intersections.

\subsubsection{Powered two wheeler oncoming vehicle information system (PTW2V)}

The system informs both the equipped car/truck/bus driver and equipped PTW rider of each other's presence if they are seen to be on a collision trajectory. Equipped vehicles (cars, buses, trucks) and PTWs wirelessly exchange information, such as position and speed. Both parties are warned of each other's presence only if they are seen to be on a collision trajectory, not in harmless situations to avoid over-informing and possibly annoying the recipient. Both are warned of an imminent collision, so both can act to prevent the accident or at least reduce speed to mitigate the consequences. The system aims to prevent accidents between cars/trucks/buses and PTWs, especially at intersections.

\subsubsection{VRU beacon system (VBS)}

The VBS consists of a tag or device carried by a VRU that sends out a signal, which is subsequently detected by a receiving device installed in vehicles. This vehicle system assesses the possibility of a collision based on the estimated trajectory of the VRU. The driver is then warned about a potential collision without any active intervention. The system is targeted to prevent accidents with all VRUs including pedestrians, cyclists, moped riders and motorcyclists. The VBS detects not only persons/vehicles in front, but also those at the rear and side of the vehicle. The VBS system is also able to detect possible collisions when there is no line of sight between vehicle and VRU. Scenarios that the VBS is mainly addressing are critical situations in urban areas, where motorised traffic travels at speeds of up to $50 \mathrm{~km} / \mathrm{h}$ and where obstructed views or unexpected behaviour of VRUs can potentially lead to conflicts. The system aims to prevent accidents between cars/trucks/buses and VRUs.

\section{Method}

\subsection{Impact assessment method}

An ex-ante method suggested by Kulmala [10] was further developed and applied here to assess the safety impacts of ITS designed specifically for VRUs. The method is described in more detail by Malone et al. [13]. The method follows the generally accepted theoretical background according to which traffic safety has three dimensions: (1) exposure, (2) risk of a collision taking place during a trip, and (3) consequences [14]. The method proposed by Kulmala [10] exploits a set of nine mechanisms through which ITS systems can affect road 
user behaviour and thereby road safety, to ascertain that all possible impacts (both positive and negative impacts on road safety; direct, indirect and unintended effects of systems) will be covered, and no effects counted twice. The nine mechanisms were modified by Malone et al. [13] to be more focused on VRUs, changes in their behaviour, and situations they face in traffic. The mechanisms, which are described in more detail elsewhere [13], are:

- Mechanism 1: Direct modification of the task of road users by giving information, advice, and assistance or taking over part of the task

- Mechanism 2: Direct influence by roadside systems mainly by giving information and advice

- Mechanism 3: Indirect modification of user behaviour in many, largely unknown ways

- Mechanism 4: Indirect modification of non-user behaviour

- Mechanism 5: Modification of interaction between users and non-users

- Mechanism 6: Modification of road user exposure by for example information, recommendation, restrictions, debiting or increased comfort in car driving, Powered Two Wheeler (PTW) riding, cycling or walking

- Mechanism 7: Modification of modal choice by for example demand restraints (area access restriction, road pricing, area parking strategies), supply control by modal interchange and other public transport management measures, and travel information systems

- Mechanism 8: Modification of route choice by route diversions, route guidance systems, dynamic route information systems, and hazard warning systems monitoring incidents

- Mechanism 9: Modification of accident consequences by intelligent injury severity reducing systems at crashes, by quick and accurate crash reporting and call for rescue, and by reduced rescue time.

As highlighted by Kulmala [10] the indirect modification of user behaviour is more long-term than the very direct, short-term reactions to the system in mechanisms 1 and 2. Long-term behavioural adaptation may appear in many different ways (for example, by reallocation of attention resources or by change of expectation of the behaviour of other road users). This behavioural adaptation may often be due to delegation of responsibility of the driving or riding task partly or totally to the system, which the drivers or riders have learnt to rely on.

The process of estimating the safety impacts of the selected ITS systems started with a literature review, followed by determination of the relevant impact mechanisms for PTW riders. The magnitude of the effect of each mechanism was assessed by experts based partly on the literature review and partly on common expertise on expected changes in road user behaviour due to the use of systems. The estimates on the effects of the selected ITS on PTW rider safety within the EU28 were calculated by assuming different penetration rates for the systems. The CARE accident database was used to scale up the results to the European level. The ERiC (European Risk Calculation) tool was utilised to facilitate the calculations. The accident data in the ERiC is divided into different categories (urban/rural, intersection/links etc.), which enables the use of different effect estimates for each category.

The method proposed by Kulmala has been applied in numerous previous projects to assess the traffic safety impacts of ITS for cars [15-20]. Because the ITS systems under assessment in our paper are not yet deployed, the possibility to validate the method lies in the future. The assumptions on the possible effects of ITS systems that we made during the assessment have been made transparent so that our findings can be compared with earlier and future assessments and validation of the results. Future validation could include field operational tests focusing on measuring road user behaviour. An example is given elsewhere [20].

\subsection{Data}

The CARE database was chosen for the analysis, as it contains details of accidents on a EU-wide level. Whilst the broad nature of the database is clearly beneficial, CARE is limited in some respects. For example, some EU Member States joined the database agreements later than others, which makes it more difficult to compare data across years due to lack of consistency. Furthermore, new Member States also means more accidentsor 'cases'-and such addition of data impacts the results by potentially indicating that an accident problem is worse than it actually is (addition of Member State data increases absolute numbers of individual collision types). Then, there is variability across Member States in the quality of accident data entered into the database, with some countries providing highly detailed and accurate data while others have many 'unknowns' due to inaccurate recording of key accident circumstances such as injury severity, age of crash participants and other factors from the scene of the accident. Finally, the database suffers from underreporting, as detailed below. Despite these shortcomings, CARE was selected for analysis because it is the best EU-wide source of accident data available. To overcome some of the above weaknesses, and to generate background variable data, the countries were grouped in three clusters based on the prevalent safety situation in each country. The clusters were 
formed using the latest road safety and VRU safetyrelated statistics. Countries with a similar safety situation, i.e. low, medium and high safety situation, were included in the same cluster. For countries and criteria where no detailed information was available in the CARE database on background variables such as road type, weather conditions, lighting conditions, location and age (or when the values were not considered reliable), the average values from the cluster to which the country belongs were used.

Total fatality and injury numbers used in the calculations are listed in Table 1. The estimation of accident numbers for 2020 and 2030 is described in chapter 3.3.

Total fatality figures for 2012 used in the impact assessment calculations for the EU-28 were taken from the Statistical Pocketbook [21]. The pocketbook does not include information on the number of injuries per se, only the number of injury accidents. We therefore compiled total numbers of injuries from the CARE database.

The more detailed information on fatalities and injuries for the EU-28 were gathered from the statistics of the CARE database for the year 2012. No accident data for 2012 was available for Belgium, Bulgaria, Estonia, Lithuania, Malta, Slovakia and Sweden, thus the latest available CARE data was used for those countries instead. For Lithuania the total numbers of fatalities and injuries in 2012 were taken from their national statistics.

It should be noted that the CARE data cannot be assumed to be complete. Underreporting is a significant and little-understood problem, especially for singlevehicle accidents of VRUs and injury accidents. Generally speaking, the issue is that the police do not record every injury accident, in addition to which the reporting

Table 1 Total number of moped, motorcycle and all road fatalities and injuries in the EU-28 used in the calculations. The 2012 data are obtained from the CARE database (injuries) and the EU statistical pocketbook (fatalities). The accident numbers for 2020 and 2030 are estimations based on the identified accident trends, see [43] for more details

\begin{tabular}{llll}
\hline & 2012 & 2020 & 2030 \\
\hline Single moped fatalities & 218 & 120 & 64 \\
Moped-vehicle fatalities & 705 & 388 & 208 \\
Single motorcycle fatalities & 1254 & 962 & 698 \\
Motorcycle-vehicle fatalities & 2877 & 2208 & 1316 \\
Single moped injuries & 21,225 & 13,114 & 7638 \\
Moped-vehicle injuries & 66,072 & 41,457 & 24,704 \\
Single motorcycle injuries & 32,379 & 25,994 & 20,111 \\
Motorcycle-vehicle injuries & 110,468 & 89,903 & 70,661 \\
All road fatalities & 28,126 & 16,429 & 8573 \\
All road injuries & $1,429,888$ & $1,085,888$ & 796,262 \\
\hline
\end{tabular}

rate is biased towards more severe accidents or those that cause traffic disruption. Actual reporting rates are not known for most EU countries. Therefore, these numbers should be treated with caution.

\subsection{Procedure}

The safety impact assessment method, described in the previous section, was applied in the VRUITS project to a set of 10 ITS. An overview of the results for the 10 ITS has been provided by Silla et al. [22]. This paper provides a more detailed assessment results regarding the three PTW related ITS.

The safety impact assessment method adopted by Malone et al. [13] and applied here followed the steps and applied the calculation tool reported by Kulmala [10] and is presented in Fig. 2. More details on the procedure for arriving at the results are provided by Silla et al. [22].

\section{System descriptions}

First, a detailed description was written for each system to ensure that everyone involved in the assessment process had a clear and confluent grasp of system's functioning, technical restrictions, projected user reactions and anticipated effects on safety.

\section{Description of effects}

During this step, the relevant safety mechanisms were selected for each studied ITS, and the expected changes in driver and VRU behaviour were described and documented for each mechanism based on the extant literature and other available evidence.

3. Estimation of effects by mechanism (mechanisms 15, 9)

Here, the safety mechanism effects were described as \% increase/decrease of relevant accidents from the baseline. An iterative approach was used for estimation of the effects, especially those for which no literature was found, by cross-checking and validating them among experts both within the study and from outside it [22].

4. Exposure effects

The results of a separate mobility assessment study [23] were applied regarding mechanisms $6-8$. The estimated effects on moped and motorcyclist exposure were converted to safety effects based on the exponential model and values found from earlier studies. These studies found a "safety in numbers" effect, i.e. the number of accidents increases less than proportionally to traffic volume (see e.g. [24]). For cyclists the meta-analysis of Elvik and Björnskau [24] includes four studies concerning road links. One Swedish, one Danish and two Dutch studies with estimates of $0.35,0.67,0.26$ and 0.44 


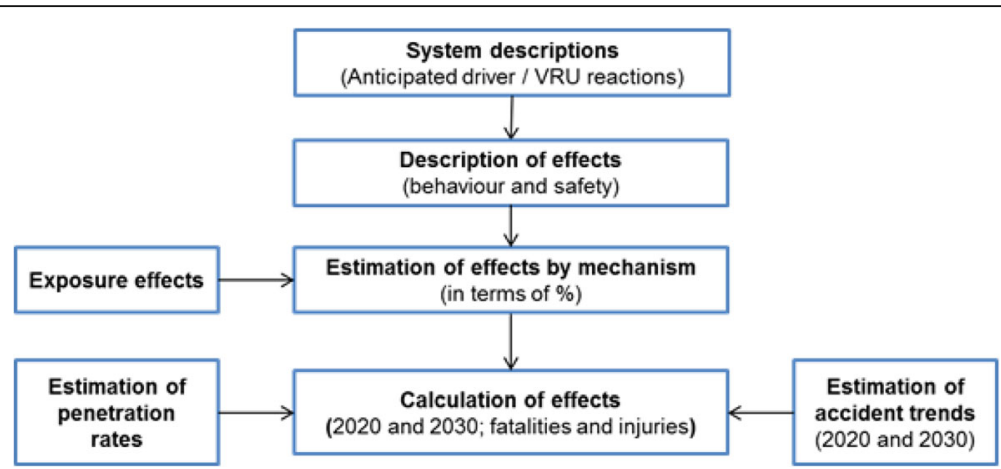

Fig. 2 Description of the overall safety impact analysis method

respectively. The average is 0.43 . Jacobsen [25] modelled fatality risk for cyclists in 14 European countries. So the sample is quite representative for EU28 compared to other studies available. His estimate of the exponent is 0.40 . The result of Elvik and Bjornskau [24] suggest that variation is larger for cyclists than for pedestrians. One likely explanation is that cycle infrastructure is very different in different EU countries. Therefore the estimate of the exponent concerning cyclists is presented with only one decimal i.e. 0.4. In VRUITS project this same exponent was used for mopeds. For motorcyclists only one reference was found [26]. Marizwan et al. [26] estimated the exponent for motorcycle flow (ADT) to be 0.404 for Malaysia. In previous EU impact assessment projects the exponent has been set to 1 (e.g. [15-17]). The mean value between the two assessments was chosen i.e. 0.7.

The effects of modal change were only included for VRUs, as those between other vehicles and public transport were considered to be insignificant.

5. Estimation of penetration rates

Penetration rates for the different systems were estimated as low, medium and high range from questionnaires sent to stakeholders and from feedback given during a workshop [16] (Table 2). The penetration rates used in the assessment for PTW2V and VBS were calculated by multiplying the vehicle penetration rates with the penetration rate of PTW. In case of INS the infrastructure penetration rate used in the calculations was higher than the one presented in Table 2. This assumption was made based on Kulmala et al. [17] who argue that the deployment of the infrastructure systems will start with the intersections with the highest numbers of accidents. Therefore, the infrastructure penetration rates were modified according to the estimates of presented by Kulmala et al. [17] which, for example, assume that $0.1 \%$ of the equipped intersections account for $3 \%$ of all intersection collisions and 1\% of the equipped intersection 10\% of intersection collisions. in 2020. After the modification the vehicle penetration rates were multiplied with infrastructure penetration rates similarly to PTW2V and VBS.

6. Estimation of accident trends for each cluster Hancox et al. [27] carried out a regression analysis of accident numbers obtained from the CARE database for the period 2002-2012 (assuming no system deployment) to predict the number of accidents for 2020 and 2030. The safety trends were organised by country cluster and accident severity ('fatal' or 'injured'). The analysis included establishment of the ratio of accidents for 2020 and 2030 for every accident that occurred in 2012 (Table 3).

Table 2 Estimate of implementation rates for the selected ITS applications for 2020 and 2030

\begin{tabular}{|c|c|c|c|c|c|c|c|}
\hline \multirow[t]{2}{*}{ System } & \multirow[t]{2}{*}{ Parameter } & \multicolumn{3}{|l|}{2020} & \multicolumn{3}{|l|}{2030} \\
\hline & & Low & Med & High & Low & Med & High \\
\hline \multirow[t]{2}{*}{ INS } & $\%$ of signalised intersections in urban areas & $0 \%$ & $0.4 \%$ & $1.5 \%$ & $2.0 \%$ & $4.0 \%$ & $5.0 \%$ \\
\hline & $\%$ of vehicles (with C-ITS equipment) & $5.0 \%$ & $10.0 \%$ & $13.0 \%$ & $15.0 \%$ & $30.0 \%$ & $40.0 \%$ \\
\hline \multirow[t]{2}{*}{ PTW2V } & $\%$ of PTWs equipped & $0.8 \%$ & $4.5 \%$ & $12.0 \%$ & $5.0 \%$ & $28.0 \%$ & $36.0 \%$ \\
\hline & $\%$ of vehicles (with C-ITS equipment) & $5.0 \%$ & $10.0 \%$ & $13.0 \%$ & $15.0 \%$ & $30.0 \%$ & $40.0 \%$ \\
\hline \multirow[t]{2}{*}{ VBS } & $\%$ of PTWs equipped & $0.8 \%$ & $5.0 \%$ & $13.0 \%$ & $5.0 \%$ & $32.0 \%$ & $40.0 \%$ \\
\hline & $\%$ of vehicles (with C-ITS equipment) & $5.0 \%$ & $10.0 \%$ & $13.0 \%$ & $15.0 \%$ & $30.0 \%$ & $40.0 \%$ \\
\hline
\end{tabular}


Table 3 Ratio of predicted 2020 and 2030 moped rider and motorcyclist accidents to every 1 moped rider / motorcyclist accident in 2012

\begin{tabular}{|c|c|c|c|c|}
\hline \multirow[t]{2}{*}{ Year } & \multicolumn{4}{|c|}{ Moped riders } \\
\hline & Cluster1 & Cluster2 & Cluster3 & Average \\
\hline \multicolumn{5}{|c|}{ Ratio to number of fatalities (number of accidents) } \\
\hline 2012 & 1.000 & 1.000 & 1.000 & 1.000 \\
\hline 2020 & 0.415 & 0.472 & 0.823 & 0.546 \\
\hline 2030 & 0.137 & 0.185 & 0.647 & 0.288 \\
\hline \multicolumn{5}{|c|}{ Ratio to number of injuries (number of serious and slight accidents) } \\
\hline 2012 & 1.000 & 1.000 & 1.000 & 1.000 \\
\hline 2020 & 0.643 & 0.584 & 0.959 & 0.655 \\
\hline 2030 & 0.373 & 0.309 & 0.993 & 0.415 \\
\hline \multirow[t]{2}{*}{ Year } & \multicolumn{4}{|c|}{ Motorcyclists } \\
\hline & Cluster1 & Cluster2 & Cluster3 & Average \\
\hline \multicolumn{5}{|c|}{ Ratio to number of fatalities (number of accidents) } \\
\hline 2012 & 1.000 & 1.000 & 1.000 & 1.000 \\
\hline 2020 & 0.694 & 0.829 & 0.736 & 0.769 \\
\hline 2030 & 0.440 & 0.656 & 0.503 & 0.559 \\
\hline \multicolumn{5}{|c|}{ Ratio to number of injuries (number of serious and slight accidents) } \\
\hline 2012 & 1.000 & 1.000 & 1.000 & 1.000 \\
\hline 2020 & 0.882 & 0.741 & 0.828 & 0.846 \\
\hline 2030 & 0.756 & 0.517 & 0.655 & 0.694 \\
\hline
\end{tabular}

\section{Calculation of effects}

The effect estimates per mechanism (from steps 3 and 4) were used to calculate the overall low, medium and high estimate on the effect of the system. These effect estimates were applied to the EU-28 road accident data, so that the distribution of the main classifying variable (collision type) weighted the estimate. For example, it could be that the investigated ITS was assumed to be effective in preventing fatalities and injuries in only one specific collision category (e.g. motorcycle accidents). For some other ITS, different collision types might be considered. In weighting, the effect estimate indicated in percent changes was multiplied by the share (\%) of relevant accidents. If, for example, a system was estimated to prevent $30 \%$ multi vehicle accidents involving motorcycles and $9 \%$ of single vehicle motorcycle accidents, the overall effect was determined by multiplying the share of relevant accidents by these effect estimates, and summing the results. In the example, this would give for the overall estimate the value " $30 \%$ multiplied by the share of multi vehicle accidents involving motorcycles $+9 \%$ multiplied by the share of single vehicle motorcycle accidents".
The overall effects by mechanism were translated into an overall effect of the system on all road fatalities/injuries at $100 \%$ penetration rate according to an illustrative example shown in Table 4. First, the estimates given in percentages were converted to coefficients of efficiency (e.g. a decrease of accidents by $10 \%$ means that the target group of accident is multiplied by coefficient 0.90 ). Secondly, the total effect was computed by multiplying the coefficients for each mechanism and giving this total effect as percentage, on the row labelled "Total medium effect". This simplified example considers only 3 mechanisms and 1 accident type. In reality, all mechanisms (with nonzero effects) were taken into account.

The estimated non-usage of systems (e.g. due to annoyance) was considered in the calculations together with the penetration rate estimates, as factors reducing the effect. In the example, this is shown on the row labelled "Total medium effect with $80 \%$ usage" for the non-usage, and on the row "Total medium effect with $60 \%$ penetration rate" for the penetration rate.

The impact of the system in number of fatalities was calculated by multiplying the overall effect expressed as a percentage of all road fatalities that will be saved or created by the total number of all road fatalities. The safety effects for $100 \%$ penetration rate (all relevant road users, vehicles and infrastructure were assumed to be equipped with the system) were calculated by exploiting the 2012 accident levels. The safety effects for future scenarios (2020 and 2030) were calculated by multiplying the effect of the system by the estimated number of fatalities of a particular scenario to arrive at the total number of fatalities saved for that scenario. A similar calculation was done for injuries, with different values for the effects. A calculation tool, adapted from the tool of Kulmala [10] for structuring the accident data and effect estimates, was used to obtain the changes in the number of fatalities and injuries per system in the EU-28.

\section{Results of the safety impact assessment}

The first three subchapters (4.1-4.3) describe the assumptions made in our safety impact assessment calculations for each system. The final subchapter (4.4) presents the results of the calculations. The results presented in this paper cover only the safety benefits obtained for PTW riders.

\subsection{Intersection safety}

The system can address all intersection accidents that involve a PTW rider and a light or heavy vehicle, where (1) the PTW rider is not hit from behind, (2) inattention played a role, and (3) the system is effective, that is, the driver or PTW rider is made aware of the danger by the warning and is able to react properly. If a PTW rider is 
Table 4 An illustrative example of calculation of the total effect on fatalities based on percent coefficients

\begin{tabular}{llll}
\hline Mechanism & Effect on fatalities & Effect, \% & Coefficient of efficiency \\
\hline Mechanism 1 & Decreases & $-10 \%$ & 0.90 \\
Mechanism 3 & Increases & $+0.5 \%$ & 1.005 \\
Mechanism 6 & Increases & $+2 \%$ & 1.02 \\
Total medium effect & & $-8 \% \leftarrow\left(0.90^{*} 1.005^{*} 1.02=0.92\right)$ & \\
Total medium effect with 80\% usage & & $-8 \% \%^{*} 80 \%=-6.4 \%$ & $-6.4 \%{ }^{*} 60 \%=-3.8 \%$ \\
Total medium effect with 60\% penetration rate & & \\
\hline
\end{tabular}

hit from behind, then that rider is generally not able to prevent the accident. For the Netherlands, the fraction of accidents where the PTW rider is not hit from behind is $99 \%$ for both fatalities and injuries. Since no better statistics are available, it is assumed that the same fractions hold for the EU. The EU-wide CARE database shows that $41 \%$ of fatal accidents and $46 \%$ of injury accidents involving mopeds with vehicles occur at intersections, compared with $41 \%$ and $54 \%$ respectively for motorcycles with vehicles.

Inattention is assumed to contribute to $30-50 \%$ of accidents. Furthermore, the system is only effective if the driver is not incapacitated, which leads to the assumption that $90 \%$ of fatalities and $94 \%$ of injuries are relevant for this system.

The effectiveness is estimated as $50-60 \%$ (the road user is made aware of the danger and system is expected to be effective), based on results from the eIMPACT [15], PReVAL [16] and Intersafe 2 projects [17, 28]. These studies looked at a similar system, the main difference being that the VRU is not warned. The system effect could be negatively impacted by overreliance, distraction, annoyance or lack of trust. eIMPACT and PReVAL both estimate a reduction of the effect of the system. eIMPACT estimates that speed at intersections will increase by $1 \%$, leading to an increase in fatalities and injuries at intersections by $2 \%$. PReVAL estimates an increase of fatalities and injuries by $1.5 \%$ due to overreliance. Assuming that the vehicle speed will increase by $0-2 \%$ (in line with the eIMPACT assumption), there is a $0-6 \%$ increase of intersection accidents due to overreliance (taking into account that VRUs are more vulnerable than vehicle passengers). For all other factors, a zero effect is assumed. In particular, usage is assessed to be $100 \%$.

If the system warns early enough, system users become aware of the danger of conflict and consequently make their intentions clear (e.g. by turning lights), which is estimated to decrease all intersection crashes by $0-0.5 \%$. Wilmink et al. [15] estimate this effect as a reduction of $0.5 \%$ of all intersection crashes, and this estimate is adopted here as a maximal effect.

The system is estimated to have a minor effect on the mobility of pedestrians and cyclists. No effect was expected for the mobility of moped riders or motorcyclists.

\subsection{Powered two wheeler oncoming information system}

If the system is in use, both PTW riders and car drivers are likely to be more aware of an upcoming hazard, lowering their speed or altering their steering response through being informed that another road user is present, thus reducing the accident risk.

Evidence regarding the nature and circumstances of PTW accidents comes from several sources. In the Motorcycle Accident In-Depth Study, or MAIDS [29], in-depth accident data covering 921 motorcycle accidents in five European countries suggested that some $60 \%$ of motorcycle accidents involve collisions with passenger cars-exactly the types of collisions that the Powered Two-Wheeler On-coming Information System aims to prevent. According to the MAIDS data, over a third of these accidents (35\%) pointed to two main accident configurations: accidents at an intersection, where the colliding vehicles were following perpendicular paths, and left-turn accidents (right-turn in the UK and Ireland), where two vehicles were approaching the intersection from opposite directions and one vehicle cut across the path of the oncoming motorcycle. Similarly, Reed \& Morris [30] found that of 283 fatal PTW accidents, $46 \%$ occurred at an intersection whereas $54 \%$ occurred away from an intersection. These figures conform to findings from the EU CARE database used in our calculations, which show that $41 \%$ of fatal accidents and $46 \%$ of injury accidents involving mopeds with vehicles occur at intersections, compared with $41 \%$ and $54 \%$ respectively for motorcycles with vehicles also at intersections.

Furthermore, Phan et al. [31] found that for PTWs, $33 \%$ of accidents occurred due to inattention. As the system draws attention to impending accidents, this figure was included in the quantitative assessment, as the system was expected to lower the number of PTW accidents caused by inattention. However, we also considered that just because a system alerts drivers/riders to an impending accident, this does not mean they will always take the necessary action. Thus, an estimate of $94-100 \%$ of drivers/riders responding to the warnings was assumed in the calculations.

There could also be some indirect negative effects of introducing this system. First there is the issue of risk 
compensation; drivers of both cars and PTWs may drive faster, assuming that the system will inform them in good time of any impending danger. This has been demonstrated for other technological systems such as vision enhancement [32] and adaptive cruise control [33-36]. However, this was not considered in our calculations. One factor that could significantly reduce the effectiveness of the system is annoyance, causing users to disable it. If it were optimally designed to alert drivers only to imminent crashes this would not be an issue, but such a perfectly working system is highly improbable. If the system gives false warnings and alerts drivers to PTWs that are simply near the car and not on a collision course with it, the system is far more likely to be turned off. It is estimated this would reduce effectiveness by a minimum of $20 \%$ to maximum of $50 \%$. The $20 \%$ figure most likely applies to motorways or rural roads, on which there are fewer motorcycles in most European countries. The 50\% applies more to urban environments, which have a higher prevalence of mopeds and motorcycles in closer proximity to cars. Possibly a PTW2V system could offer added comfort to a PTW rider by reducing the workload, lessening anxiety, and giving assurance that the rider will not be hit by an oncoming vehicle and will better be able to react to a developing situation. This could result in more or longer pleasure rides.

There is no literature to date that has investigated the effect of driver assistance systems on mileage. However, Jamson \& Chorlton [37], when investigating what motivates motorcycle usage, found that, 'The nature of motorcycling ... for some has become a leisure activity, with the motorcycle being more of an accessory than a means of transport.' This suggests that the usage of motorcycles may be more sensitive to such changes than most forms of transport, as a result of it often being used for pleasure as opposed to commuting or business. This increase in comfort or perceived safety could therefore possibly have a large effect on usage rate.

As a result, the system is estimated to lead to more pleasure trips and even to an actual switch in modality towards moped and motorcycle usage for everyday commuting. Specifically, the road exposure of moped riders is expected to increase by $0.4-0.7 \%$ with use of the system and that of motorcyclists by $0-1.4 \%$. In addition to changes related to direct exposure effects (new and longer trips), impacts are also expected on modal change and are estimated to increase moped rider exposure by $0-1.3 \%$ and motorcyclist exposure by $0-0.3 \%$.

\subsection{Vulnerable road user beacon system}

The system was estimated to prevent accidents between vehicles and PTWs occurring in urban areas. The EUwide CARE database shows that $52 \%$ of fatal mopedvehicle accidents and $84 \%$ of moped-vehicle accidents resulting in injuries occur in urban areas. The corresponding shares for motorcycle-vehicle accidents were $40 \%$ regarding fatalities and $73 \%$ regarding accidents resulting in injuries. In addition, according to Phan et al. [31], 33\% of PTW accidents occur as a result of nondetection or inattention of the car driver. In addition, it is assumed that $85 \%-90 \%$ of equipped car/truck/bus drivers will actually obey the warning and react in time, and thus be able to avoid a collision.

Due to the warnings provided by the system, annoyance due to high detection levels of VRUs, especially in urban environments, where numerous pedestrians and bicyclists are in the vicinity of the vehicle, can have a significant impact on speed, throttle control and lateral position on the road. While annoyance can lead to an increased workload of direct system users, a tactile or audio-visual warning, despite being annoying, has not been shown to have a significant negative impact on actual accident risk. Nevertheless, increasing numbers of audio warnings/information are associated with reduced willingness to react to the provided information [38].

The potential effects of this are expected to lead to nonusage of the system in $20-30 \%$ of cases. In addition, overreliance of either a car driver or a PTW rider expecting a reaction from the other road user could lead to a negative impact in view of the system's aims. As the VBS is only actively used by the car driver, overreliance is assumed to be higher for the active user (car driver) compared to informed PTW rider, who expects to be detected. In these situations, either PTW riders or car drivers are assumed to be less vigilant due to the available system. Also in these situations, overreliance could well diminish the expected positive impacts of the system by $24-34 \%$, if either of the above negative effects applies.

The road exposure of moped riders is expected to increase by $0.3-0.8 \%$ and the road exposure of motorcyclists by $0.1 \%-0.8 \%$ with use of the system. In addition to changes related to direct exposure (new and longer trips), impacts are also expected on modal change, which is estimated to increase moped rider exposure by $0.1-0.7 \%$ and the road exposure of motorcyclists by 0 . $1 \%-0.7 \%$. These exposure changes were assumed based on studies relating to risk perception and subjective safety impacts on modal choice (see e.g. [39]). These changes was expected to be especially relevant to the group of motorcyclists which shows comparatively high levels of insecurity and a lack of subjective safety in traffic [40-42]. Therefore, the estimated increase in PTW trips is expected to result from a more optimistic safety perception of this mode of transport.

\subsection{Overall results}

According to our principle results, all of the systems we studied have a positive impact on PTW rider safety by 
preventing fatalities and injuries. When considering full penetration, the best estimates for safety would be achieved by adopting PTW2V and INS, and the poorest by the Vulnerable road user Beacon System (VBS). The estimated medium yearly reduction in percentage of all road fatalities within the EU-28 is approximately 1.0\% for PTW2V, $0.9 \%$ for INS, and $0.8 \%$ for VBS (Fig. 3). The estimated medium yearly reduction in number of PTW rider fatalities at 2012 accident levels in the EU-28 is 283 for PTW2V, 261 for INS and 216 for VBS (Fig. 4).

The forecasts for 2020 and 2030, taking into account the estimated penetration rates and accident trends, showed the highest effects for INS (nine fewer fatalities in 2030), followed by PTW2V (seven fewer fatalities in 2030) and VBS (six fewer fatalities in 2030) (Fig. 5). It should be noted that the impacts are calculated for the assumed penetration rates and have to be recalculated when more accurate measurements of penetration rates become available.

\section{Discussion and conclusions}

The objective of this study was first to identify the mechanisms of impact through which selected ITS systems influence the safety of PTW riders, and to describe their effects. Second, we aimed to estimate these impacts quantitatively once these ITS systems are fully deployed in the EU-28, including in selected future scenarios for 2020 and 2030. According to the analysis, all of the systems we looked at have a positive impact on PTW rider safety by preventing fatalities and injuries. The best effects (when considering 2012 accident data and full penetration) were offered by PTW2V and INS, and the weakest by VBS. The estimated medium annual drop in PTW rider fatalities in the EU-28 varied between 216 and 283 per system at 2012 accident levels. The strong effect of PTW2V is not surprising, since this system potentially addresses all multi-vehicle accidents. It could be even increased if higher levels of usage attained (currently $20-50 \%$ of non-usage was assumed to be caused

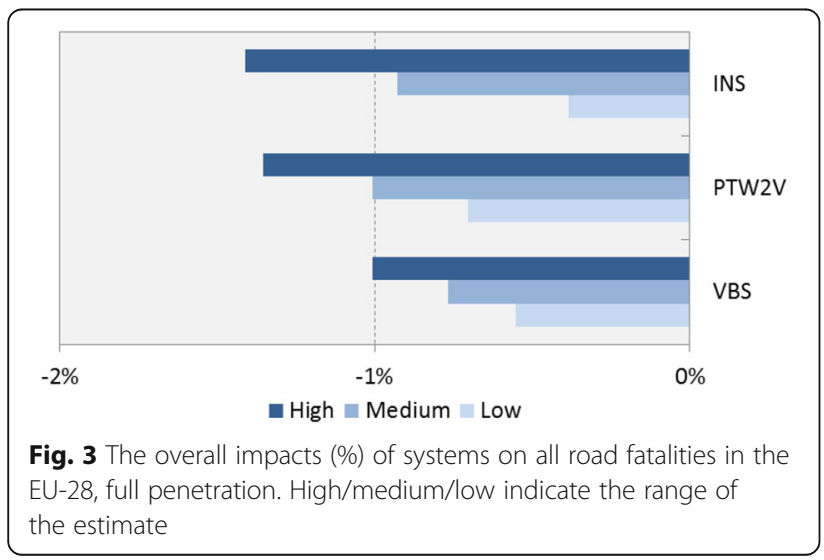

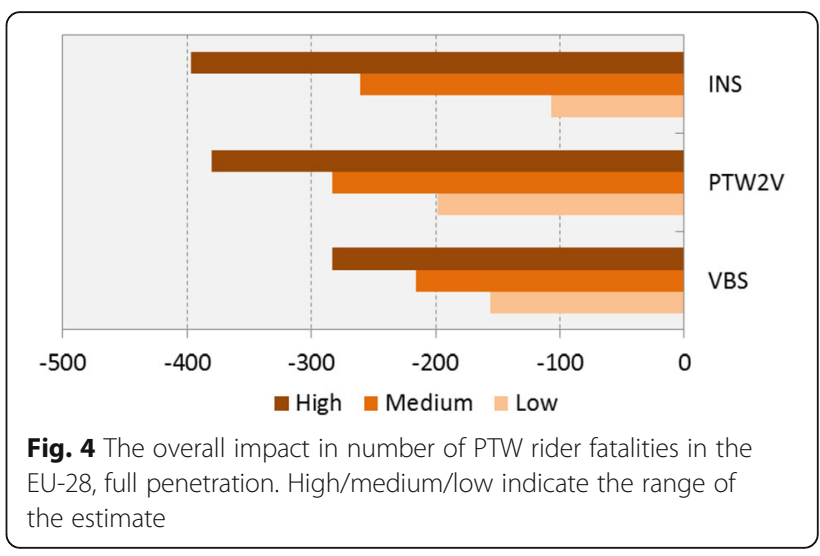

by annoyance). Furthermore, based on the results, the other two systems (INS and VBS) also show significant potential for improving the safety of PTW riders. Their effects are, however, slightly lower, mainly because INS and VBS target only specific situations - INS intersection accidents, and VBS mainly situations with obstructed views or unexpected behaviour of VRUs. However, even small safety effects can be significant when, as we assume will happen in the future, the systems are introduced as combinations or bundles, especially when individual systems in a bundle target different type of accidents.

A look-ahead to 2030 considering predicted penetration rates and accident trends provides a more realistic view of the expected effects. The results for future scenarios (2030) showed the strongest effects on fatality numbers per system in the EU-28 for intersection safety. In our assessment, the level of penetration, which was based on questionnaires and feedback from stakeholders, was assumed to be quite low. The respondents were not specifically asked to provide promoted scenarios (e.g. policy actions of the European Commission), therefore the responses can be considered conservative (businessas-usual scenarios). An additional reason for relatively low penetration rates is that all the investigated systems require that two parties are equipped with the system. In

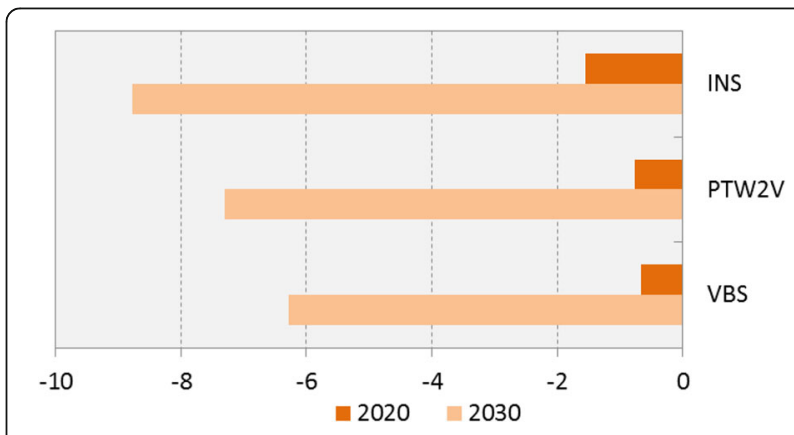

Fig. 5 The overall medium impact in number of PTW rider fatalities in the EU-28, future scenarios 
PTW2V and VBS, both PTW riders and vehicles need to be equipped with the system, and INS requires equipping both vehicles and the infrastructure. We acknowledge that even over the short-term period since the questionnaires were completed, several European Commission (e.g. the C-ITS Platform) and other promotional activities have taken place (such as the discussion on automated driving), in which case the estimated penetration rates could well be higher now.

User acceptance of ITS among PTW riders might be an issue for deployment. For example, systems designed for cars are not often suitable as such for PTW riders. Different types of sensors, visual user interfaces, integration with vehicles and standards are needed for PTWs. In addition, the cost of a system, initially designed for cars, should be better adjusted to that of a PTW. That said, implementation of ITS systems can provide added value to the PTW riding experience, for example through better road markings needed for the systems. In addition, ITS warnings of problems typical to PTW, such as slippery road surfaces, manholes, and crosswalks on curves would make the systems more desirable for PTW riders.

It is important to note that the provided estimates on safety effects consider future systems in future scenarios, which makes estimated numbers of avoided fatalities and injuries in the EU-28 somewhat uncertain. In general, this uncertainty can derive from a) estimates of safety effects (which depend on the results of expert questionnaires and findings from the literature), b) accident data (which depends on the quality of data on different accident types), c) estimated accident trends, and d) estimated penetration rates. The uncertainty was addressed by providing low, medium and high values for all the estimates related to each relevant safety mechanism. Similarly, the estimates of penetration rates included low, medium and high values. Uncertainties in accident data and accident forecasts were not addressed. The results of this paper concern only fatalities. This is because during the assessment process it became evident that the underreporting of injuries is common and the extent of this problem varies between countries. For fatalities, the data is of better quality but not perfect either.

Moreover, when interpreting the results for future scenarios, it is important to note that the impact difference between 2020 and 2030 is partly due to the current trend of year-by-year drop in number of fatalities and injuries being expected to continue into the future, which is a rather conservative assumption. This trend is subject to a wide range of safety enhancements (e.g. improvements in infrastructure, vehicles, driver and traveller training, etc.) other than the systems under consideration in this project. The consequence of this trend is that there will be fewer fatalities and injuries in 2020 and 2030, hence a system that prevents the same fraction of fatalities and injuries in 2020 as in 2030 will have lower savings in 2030 than in 2020 in absolute numbers. On the other hand, PTW ridership can increase for a number of reasons, which would influence accident trends and thus increase the number of PTW rider accidents in respect to the estimate. The trends have been determined separately for different VRU groups and cars, because the historical trend shows large differences, but further subdivision (e.g. by accident type) has been deemed unnecessary and unpractical.

In summary, the results of this study show that the ITS under study have a strong potential to improve the safety of PTW riders. It should be noted that the impacts are calculated for the assumed penetration rates and have to be recalculated when more accurate measurements of penetration rates become available. In order to realise the potential safety effects (or obtain even higher safety benefits than estimated in this study), the deployment of the most promising systems should be supported by different stakeholders and decision makers. In future, the accuracy of the estimates can be improved by better accident data (more detailed information on numbers and details of accidents, including also hospital data) and by trials to collect more information on the functioning of the systems and their effect on road user behaviour.

\section{Acknowledgements \\ The research was funded by the European Union's Seventh Framework Programme under grant agreement $n^{\circ} 321586$ (VRUITS) and participating partners.}

\section{Authors' contributions}

AS with the support of $L L$ and PR, was responsible of leading the work on assessing the safety effects of each system and providing support and comments to the assessment of effects of each system on road user behavior. In addition, AS conducted the calculations to obtain the numerical safety effects of each system. JS was responsible on identifying the penetration rates for different systems whereas $\mathrm{AM}$ and $\mathrm{GH}$ carried out the estimation on accident trends for each system. The work on the analysis of the effect of each system on road user behaviour was divided as follows: MvN lead the analysis on intersection safety, AM and GH lead the analysis on Powered Two Wheeler oncoming information system and DB led the analysis on vulnerable road user beacon system. All authors read and approved the final manuscript.

\section{Competing interests}

The authors declare that they have no competing interests.

\section{Publisher's Note}

Springer Nature remains neutral with regard to jurisdictional claims in published maps and institutional affiliations.

\section{Author details}

${ }^{1}$ VTT Technical Research Centre of Finland Ltd, P.O. Box 1000, 02044 VT, Espoo, Finland. ${ }^{2}$ TNO, PO Box 49, 2600 AA Delft, The Netherlands.

${ }^{3}$ Loughborough Design School, Loughborough University, Loughborough, Leicestershire LE11 3TU, UK. ${ }^{4}$ FACTUM Chaloupka \& Risser OG,

Danhausergasse 6/4, 1040 Vienna, Austria. 


\section{Received: 6 April 2016 Accepted: 5 April 2018}

\section{Published online: 18 May 2018}

\section{References}

1. European Road Safety Observatory (2017) Traffic safety basic facts 2016, https://ec.europa.eu/transport/road_safety/sites/roadsafety/files/pdf/ statistics/dacota/bfs2016 motomoped.pdf. Accessed 28 June 2017

2. Vademecum. (2015). Road safety in the European Union. Trends, statistics and main challenges, March 2015. This report is an internal working material summarising preliminary EU road safety information for 2014 and final detailed data as submitted by the EU Member States for the years until 2013. European Commission. http://ec.europa.eu/transport/road_safety/pdf/ vademecum_2015.pdf. Accessed 8 Mar 2016

3. Mattsson, M., Leden, L. (2015). Cognitive ergonomic models of rider crashes. State of the rider user, the vehicle and traffic environment for scenarios outside urban area. Presented at the 29th ICTCT workshop in Ashdod, Israel. October 29th-30th, 2015

4. Rosander, P. (2011). MC-förares attityder och riskmedvetenhet. http://www. Itu.se/research/subjects/Arkitektur/2.51143/MC-forares-attityder-ochriskmedvetenhet-1.78974. Accessed 8 Mar 2016

5. Christensen, J. Nordqvist, M. (2014). Extremt Beteende - främst en fråga om olovlig körning. Transportforum, Linköping, 2014. http://www.svmc. se/smc_filer/SMC\%20centralt/Rapporter/2014/Extremt_beteende.pdf. Accessed 8 Mar 2016

6. Beanland $\mathrm{V}$, Lenné $M$, Fuessl E, Oberlader M, Joshi S, Bellet T, Banet A, Rößger L, Leden L, Spyropoulou I, Yannis G, Roebroeck H, Carvalhais J, Underwood G (2013) Acceptability of rider assistive systems for powered two-wheelers. Transp Res F 19(2013):63-76

7. The Swedish Transport Administration. (2010). Improved safety for motorcycle and moped riders. Joint strategy for the period 2010-2020, version 1.0. ISSN 978-91-7467-014-1

8. Vegdirektoratet (2004) MC-sikkerhed. Udformning og drift av veg- og trafikksystemer. Håndbok 22, Oslo

9. Barmpounakis E, Vlahogianni El, Golias JC (2016) Intelligent transportation systems and powered two wheelers traffic, intelligent transportation systems. IEEE Transactions on 17(4):908-916

10. Kulmala R (2010) Ex-ante assessment of the safety effects of intelligent transport systems. Accid Anal Prev 42(2010):1359-1369

11. Scholliers, J., Bell, D, Morris, A. \& García-Meléndez, A.B. (2014). Potential of ITS to improve safety and mobility of VRUs. 10th ITS European congress, Helsinki, Finland, 16-19 June, 2014

12. Kruijff, J, Malone, K. (2014). VRUITS milestone MS2 - second interest group workshop

13. Malone K, Silla A, Johansson C, Bell D (2017) Safety, mobility and comfort assessment methodologies of intelligent transport systems for vulnerable road users. Eur Transp Res Rev 9:21

14. Nilsson G (2004) Traffic safety dimensions and the power model to describe the effect of speed and safety. Bulletin 221. Department of Technology and Society. Lund University, Sweden

15. Wilmink, I., Janssen, W., Jonkers, E., Malone, K., van Noort, M., Klunder, G., Rämä, P., Sihvola, N., Kulmala, R., Schirokoff, A., Lind, G., Benz, T., Peters, H. \& Schönebeck, S. (2008). Impact assessment of intelligent vehicle safety systems. elMPACT deliverable D4, 11 august, 2008

16. Scholliers, J., Heinig, K., Blosseville, J., Netto, M., Anttila, V., Leanderson, S., Engström, J., Ljung, M., Hendriks, F., Ploeg, J. \& Chen, J. (2007). D16.3 proposal of procedures for assessment of preventive and active safety functions. PreVENT SP deliverable

17. Kulmala, R., Leviäkangas, P., Sihvola, N., Rämä, P., Francsics, J., Hardman, E., Ball, S., Smith, B., McCrae, I., Barlow, T. \& Stevens, A. (2008). Final study report. CODIA deliverable 5

18. Wimmershoff M, Will D, Pütz A, Lach A, Schirokoff A, Pilli-Sihvola E, Le L, Zlocki A, Sihvola N, Weingart J, Stimming C, Kulmala R (2011) Test and evaluation results. Deliverable D8.2, INTERSAFE2, p 162

19. Fuerstenberg, K. \& Boehning, M. (2012). Final report. D1.2, MINIFAROS project

20. Innamaa, S., Rämä, P., Hogema, J., Kroon, L., Heinig, I., Schindhelm, R., Ojeda, L., Servel, A., Visin-tainer, F. \& Giosan, I. (2014). Safety impacts of cooperative systems. Deliverable IR4.Y of DriveC2X project

21. EC. (2014). EU transport in figures. Statistical pocketbook 2014. http://ec. europa.eu/transport/facts-fundings/statistics/doc/2014/pocketbook2014.pdf. Accessed 8 Oct 2014
22. Silla, A., Rämä, P., Leden, L., van Noort, M., de Kruijf, J., Bell, D., Morris, A., Hancox, G. \& Scholliers, J. 2017. Quantifying the effectiveness of ITS in improving safety of VRUs. IET intelligent transport systems. IET, vol. 11,3 , ss. 164-172

23. Johansson, C., Bell, D., Garcia Mendelez, A., Perez, O.M. (2014). Internal report on assessment of mobility and comfort. VRUITS internal report

24. Elvik, R. \& Bjørnskau, T. (2017). Safety-in-numbers: a systematic review and meta-analysis of evidence. Safety science 92 , special issue article: cycling safety, 274-282

25. Jacobsen PL (2003) Safety in numbers: more walkers and bicyclists, safer walking and bicycling. Injury prevention 9(3):205-209

26. Marizwan bin Abdul Manan M (2014) Factors associated with motorcyclists' safety at access points along primary roads in Malaysia. Lund University, Faculty of Engineering, Doctoral Dissertation, Lund

27. Hancox G, Morris A, Silla A, van Noort M, Bell D, Scholliers J (2015) Current and future trends in vulnerable road-user accidents in Europe - why we need ITS solutions. World congress on intelligent transport systems, 5-9 October 2015. Bordeaux, France, ITS America, ITS Asia-Pacific

28. Schirokoff A, Pilli-Sihvola E, Sihvola N (2012) Assessing the safety impacts of intersection safety systems. Proceedings TRA 2012. Procedia - Social and Behavioral Sciences 48:1515-1524

29. MAIDS. (1999). Data summarised from: http://www.wheelchair.ch/fra/moto/ images/docs/honda_cartocar.pdf. Accessed 8 Oct 2014

30. Reed S, Morris AP (2012) Characteristics of fatal single-vehicle crashes in Europe. International Journal of Crashworthiness 17(6):655-664

31. Phan, V., Regan, M., Moutreuil, M., Minton, R., Mattsson, M. \& Leden, L. (2010). Using the Driving Reliability and Error Analysis Method (DREAM) to understand Powered Two-Wheeler accident causation. International Conference on Safety and Mobility of Vulnerable Road Users: Pedestrians, Motorcyclists and Bicyclists. Jerusalem

32. Stanton N, Pinto M (2000) Behavioural compensation by drivers of a simulator when using a vision enhancement system. Ergonomics 439: 1359-1370

33. Nilsson, L. (1995). Safety effects of adaptive cruise controls in critical traffic situations. In steps forward. Intelligent transport systems world congress (no. volume 3)

34. Young MS, Stanton NA (1997) Automotive automation: investigating the impact on drivers' mental workload. Int J Cogn Ergon 1(4):325-336

35. Stanton NA, Young MS (1998) Vehicle automation and driving performance. Ergonomics 41(7):1014-1028

36. Xiong H, Boyle LN (2012) Drivers' adaptation to adaptive cruise control: examination of automatic and manual braking. Intelligent Transportation Systems, IEEE Transactions on 13(3):1468-1473

37. Jamson S, Chorlton K (2009) The changing nature of motorcycling: patterns of use and rider characteristics. Transport Res F: Traffic Psychol Behav 12(4):335-346

38. Walker BN, Nees MA (2011) Theory of sonification. In: Hermann T, Hunt A, Neuhoff JG (eds) The Sonification handbook, chapter 2. Logos Publishing House, Berlin, Germany, pp 9-39

39. Fyhri $A$, Hof $T$, Simonova $Z$, de Jong $M$ The influence of perceived safety and security on walking. In: Methorst R, Monterde-i-Bort H, Risser R, Sauter $D$, Tight \& Walker (eds) Pedestrians' quality needs. Final report of the COST project 358, Cheltenham: Walk21, 2010, part B.2 perceived needs, pp 49-69

40. Amundsen AH, Bjørnskau T (2003) Utrygghet og risikokompensasjon i transportsystemet. (Report No. 622). Transportøkonomisk Institutt, Oslo, Norway

41. Bjørnskau T (2004) Trygghet i transport. Oppfatninger av trygghet ved bruk av ulike transportmidler, TøI-rapport 702. Transportøkonomisk institutt, Oslo

42. Furian G, Brandstätter C, Kaiser S, Witzik A (2016) Subjective safety and risk perception. ESRA thematic report no. 5. ESRA project (European survey of road users' safety attitudes). Kuratorium für Verkehrssicherheit, Vienna, Austria

43. Hancox, G., Morris, A., Silla, A., van Noort, M., Bell, D. \& Scholliers, J. (2015). Current and future trends in vulnerable road-user accidents in Europe why we need ITS solutions. Paper accepted for presentation in ITS world conference, 5-9 October, 2015. Bordeaux, France 